\title{
Quantifying the Intrinsic Effects of Two Point Mutation Models of Pro-Pro diamino acid diamide. A First-Principle Computational Study
}

\author{
M. A. Sahai ${ }^{a,}$, B. Viskolcz ${ }^{b}$, E. F. Pai ${ }^{a, c}$ and I.G. Csizmadia ${ }^{b, d,{ }^{*}}$ \\ aDepartment of Medical Biophysics, University of Toronto, Toronto Medical Discovery Tower, \\ 101 College Street, Rm. 5-359, Toronto, Ontario, Canada, M5G 1 L7 \\ ${ }^{b}$ Department of Chemistry and Chemical Informatics, Faculty of Education, University of Szeged \\ Boldogasszony sgt. 6, Szeged, Hungary, 6725 \\ 'Department of Biochemistry and Molecular and Medical Genetics, University of Toronto, \\ Toronto, Ontario Canada, M5S 1 A8 \\ ${ }^{\mathrm{d}}$ Department of Chemistry, University of Toronto, 80 St. George St., Toronto, Ontario, Canada, M5S 3H6
}

\section{SUPPORTING INFORMATION}

Figure A. Numbering system employed for HCO-Pro-Pro- $\mathrm{NH}_{2}, \mathrm{HCO}-\mathrm{Pro}-\mathrm{Thr}-\mathrm{NH}_{2}$, and HCO-Thr-Pro- $\mathrm{NH}_{2}$ with definitions of backbone and sidechain dihedral angles.

Figure B. Schematic representation of the conformations that converged for the dipeptide HCO-Pro-Pro- $\mathrm{NH}_{2}$, listed in Tables $\mathbf{D}$ and $\mathbf{E}$ of the Supporting Information. Solid circles represent those conformations found at the RHF/3-21G level of theory while those found at the B3LYP/6-31G(d) level of theory are in the broken circles.

Figure C. Optimized conformations of the $30 \mathrm{HCO}-\mathrm{Pro}-\mathrm{Thr}-\mathrm{NH}_{2}$ conformations computed at the B3LYP/6-31G(d) level of theory.

Figure C. Schematic representation of the conformations that converged for the dipeptide HCO-Pro-Thr- $\mathrm{NH}_{2}$, listed in Table $\mathbf{H}$ of the Supporting Information, at the B3LYP/6$31 \mathrm{G}(\mathrm{d})$ level of theory. Note that no cis-cis conformers are identified in the schematic because no cis-cis conformers were retrieved from the PDB.

Figure D. Schematic representation of the conformations that converged for the dipeptide HCO-Thr-Pro- $\mathrm{NH}_{2}$, listed in Table I of the Supporting Information, at the B3LYP/6$31 \mathrm{G}(\mathrm{d})$ level of theory. Note that no cis-cis conformers are identified in the schematic because no cis-cis conformers were retrieved from the PDB.

Figure E. Optimized conformations of the $8 \mathrm{HCO}-\mathrm{Pro}-\mathrm{Pro}-\mathrm{NH}_{2}$ conformations computed at the B3LYP/6-31G(d) level of theory.

Figure G. Optimized conformations of the $34 \mathrm{HCO}-\mathrm{Thr}-\mathrm{Pro}-\mathrm{NH}_{2}$ conformations computed at the B3LYP/6-31G(d) level of theory.

Table A. Retrieved parameters from the Brookhaven Protein Database for 16 unique ProPro dipeptide sequences.

Table B. Retrieved parameters from the Brookhaven Protein Database for 36 unique ProThr dipeptide sequences. 
Table C. Retrieved parameters from the Brookhaven Protein Database for 28 unique ThrPro dipeptide sequences.

Table D. Selected Parameters of HCO-Pro-Pro- $\mathrm{NH}_{2}$ at the RHF/3-21G level of theory.

Table E. Selected Parameters of HCO-Pro-Pro- $\mathrm{NH}_{2}$ at the B3LYP/6-31G(d) level of theory.

Table F. Selected Parameters of HCO-Pro-Thr- $\mathrm{NH}_{2}$ at the RHF/3-21G level of theory.

Table G. Selected Parameters of HCO-Thr-Pro- $\mathrm{NH}_{2}$ at the RHF/3-21G level of theory.

Table H. Selected Parameters of HCO-Pro-Thr- $\mathrm{NH}_{2}$ at the B3LYP/6-31G(d) level of theory.

Table I. Selected Parameters of HCO-Thr-Pro-NH $\mathrm{NH}_{2}$ at the B3LYP/6-31G(d) level of theory.

Table J. A list of conformations that converged away from their initial geometries at the B3LYP/6-31G(d) level of theory for the three dipeptides. 


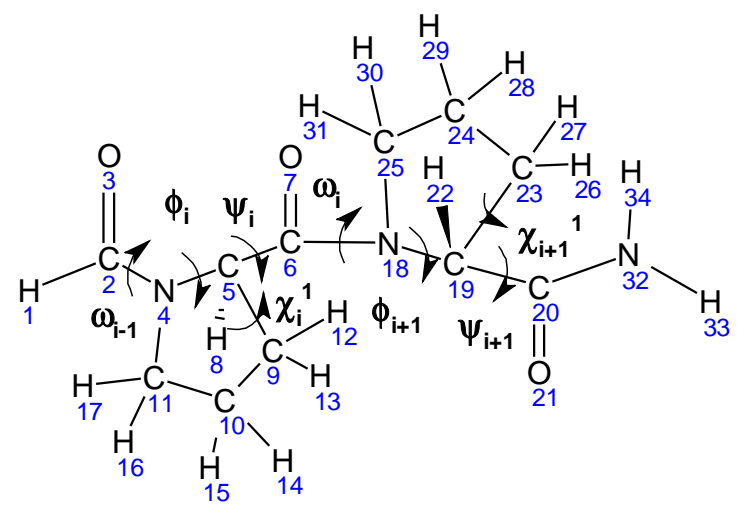

HCO-Pro-Pro-- $\mathrm{NH}_{2}$

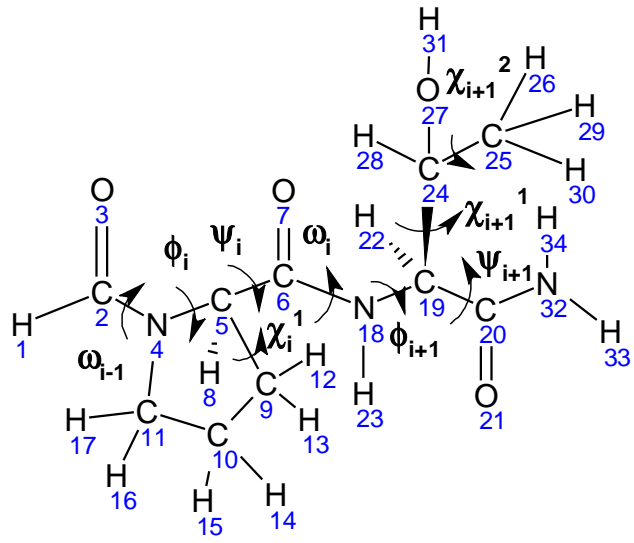

HCO-Pro-Thr- $\mathrm{NH}_{2}$

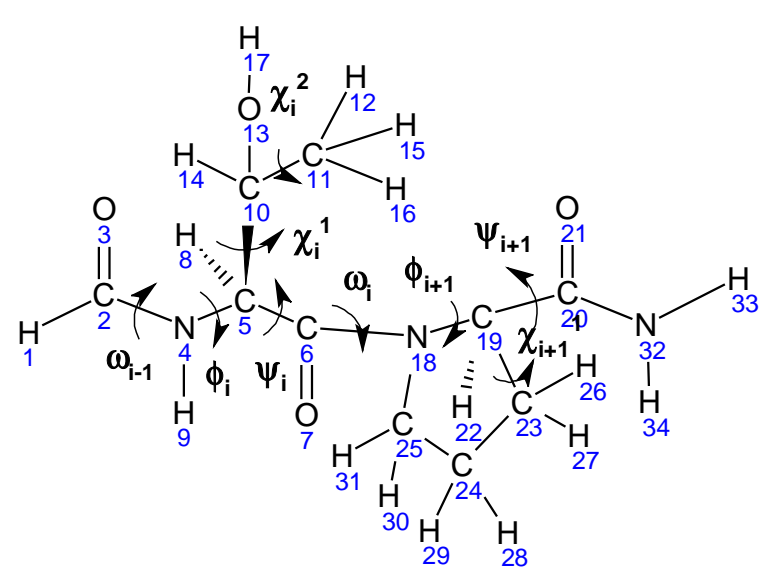

HCO-Thr-Pro-NH ${ }_{2}$

Figure A. Numbering system employed for HCO-Pro-Pro- $\mathrm{NH}_{2}, \mathrm{HCO}-\mathrm{Pro}-\mathrm{Thr}-\mathrm{NH}_{2}$, and HCO-Thr-Pro- $\mathrm{NH}_{2}$ with definitions of backbone and sidechain dihedral angles. 

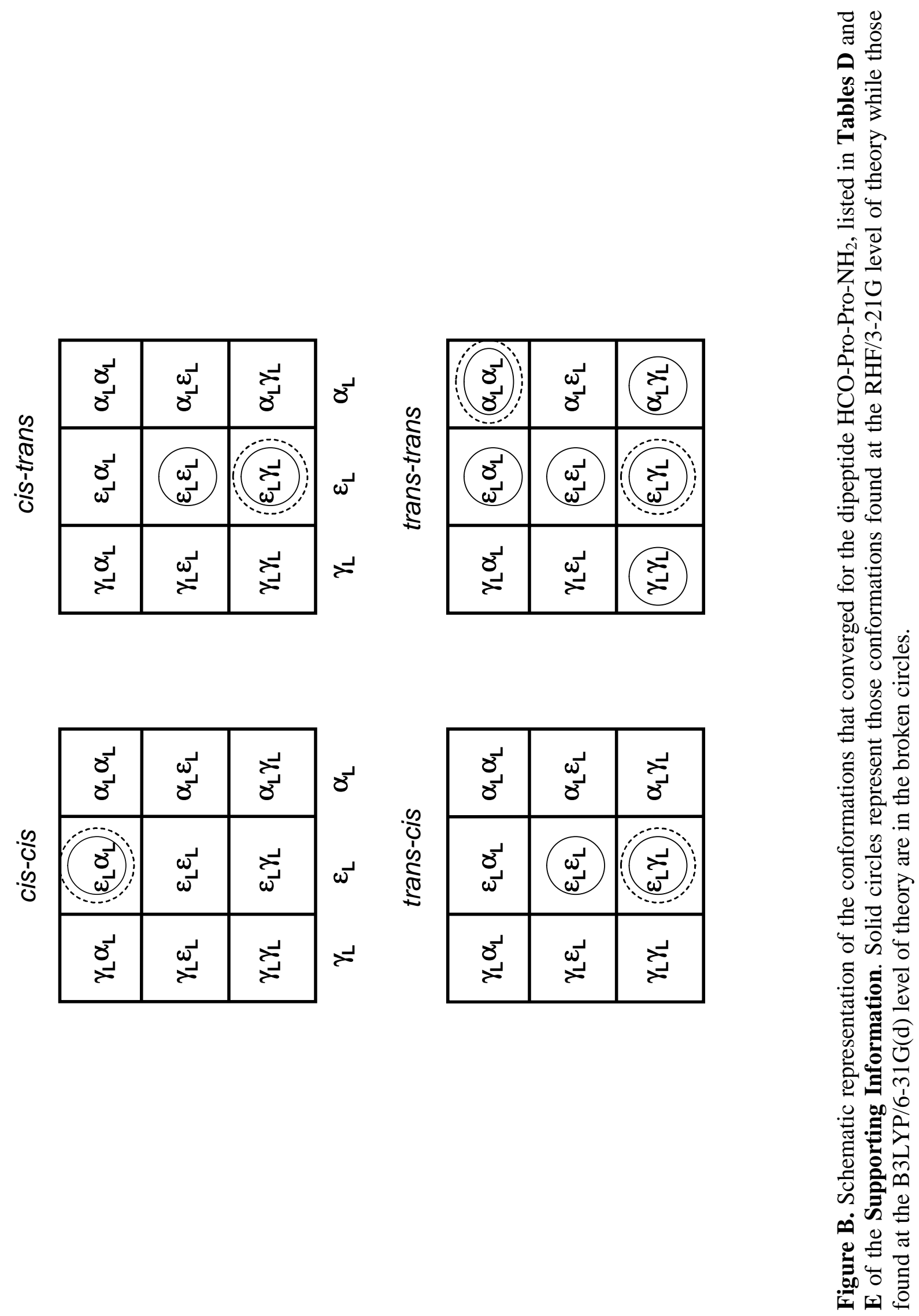


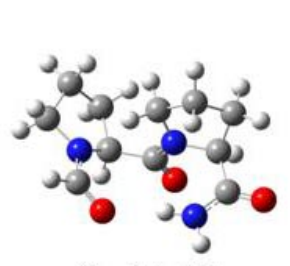

tt $\alpha_{L}[+] \alpha_{L}[+]$
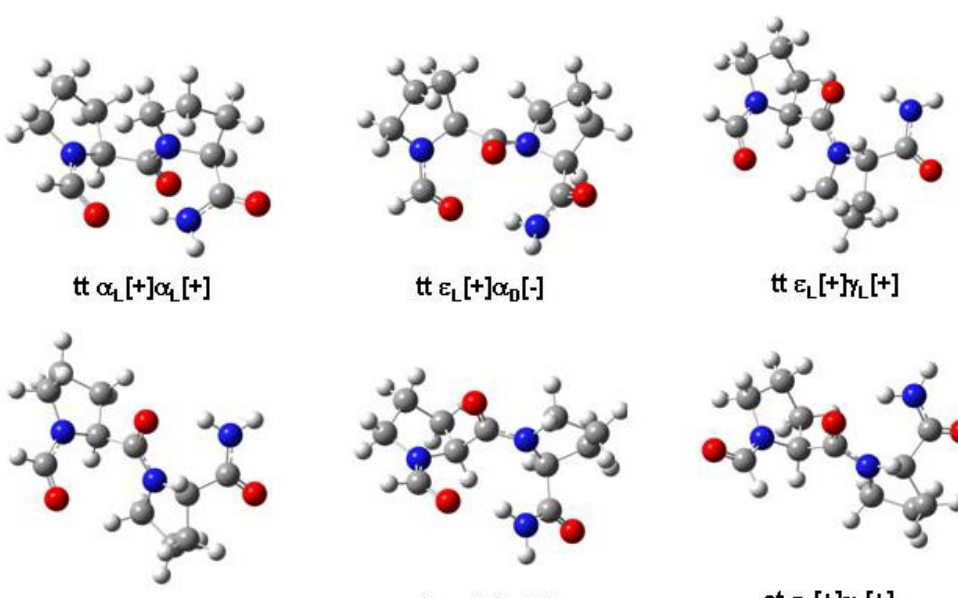

tt $\varepsilon_{\mathrm{L}}\left[-\mathrm{yy}_{\mathrm{L}}[+]\right.$

tt $\varepsilon_{\mathrm{L}}[+] \alpha_{\mathrm{D}}[-]$

tt $\varepsilon_{\mathrm{L}}\left[+\mathrm{y}_{\mathrm{L}}[+]\right.$

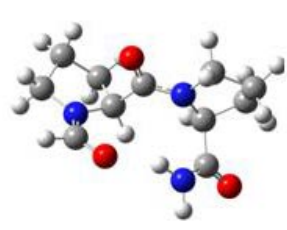

tc $\varepsilon_{\mathrm{L}}[+] \mathrm{y}_{\mathrm{L}}[+]$

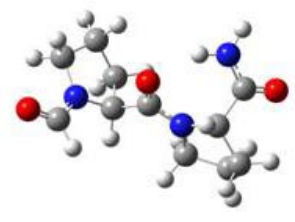

ct $\varepsilon_{\mathrm{L}}[+] \mathrm{y}_{\mathrm{L}}[+]$

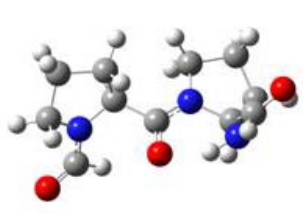

ct $\varepsilon_{L}[-] y_{L}[+]$

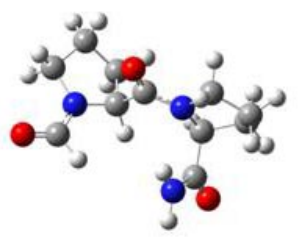

$\operatorname{cc} \varepsilon_{\mathrm{L}}[+] \alpha_{\mathrm{L}}[+]$

Figure C. Optimized conformations of the $8 \mathrm{HCO}-\mathrm{Pro}-\mathrm{Pro}-\mathrm{NH}_{2}$ conformations computed at the B3LYP/6-31G(d) level of theory. 


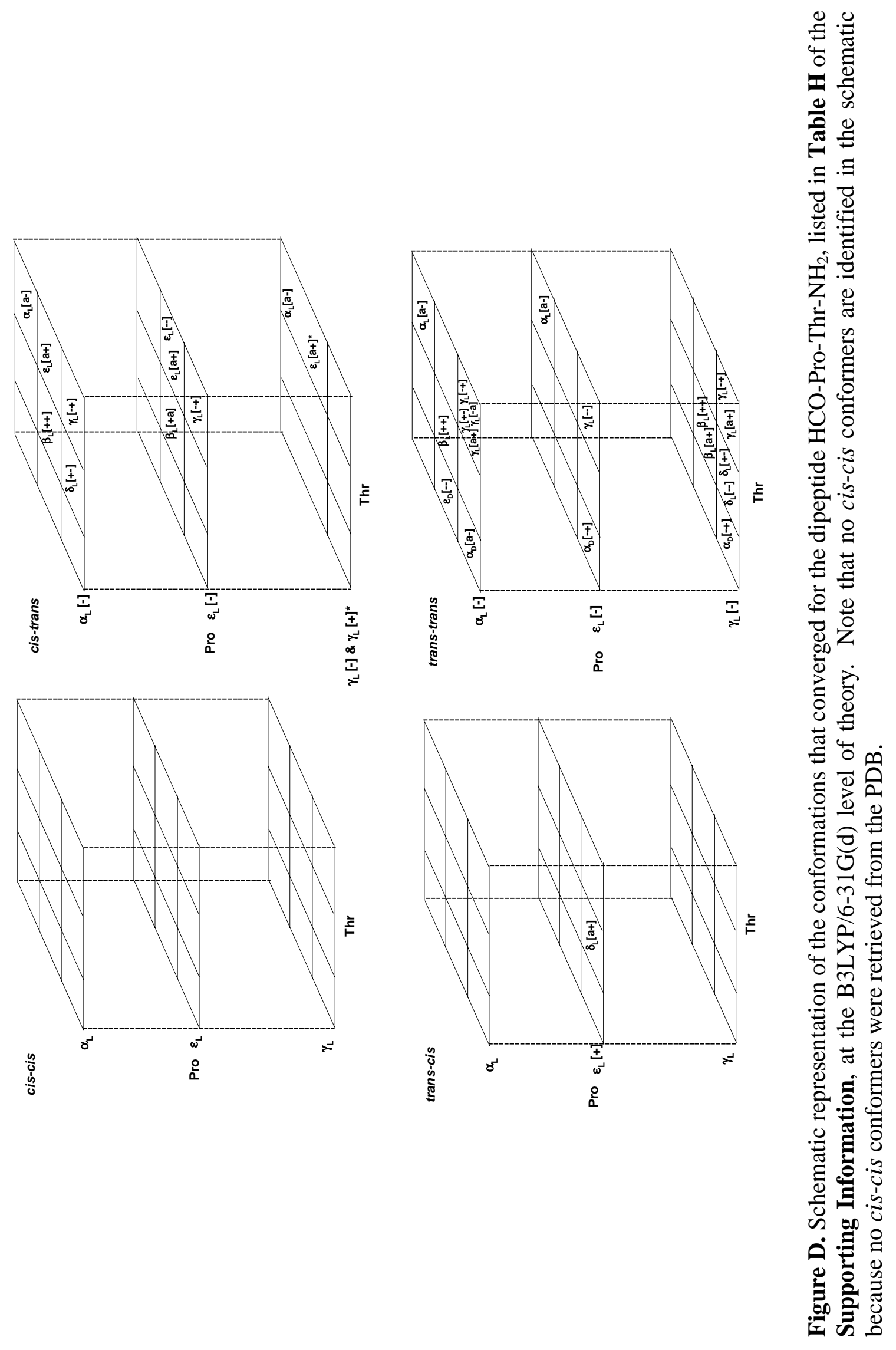




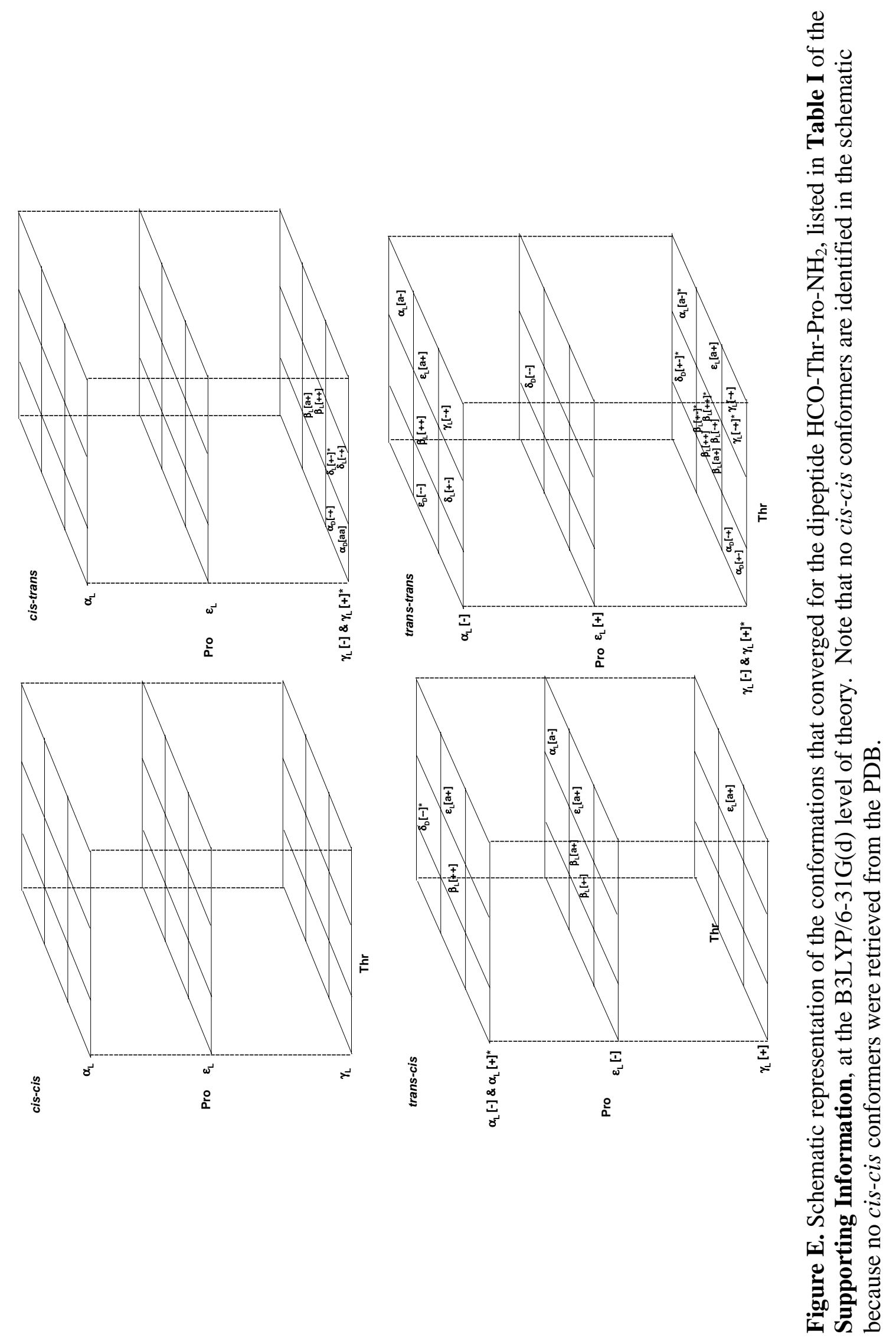




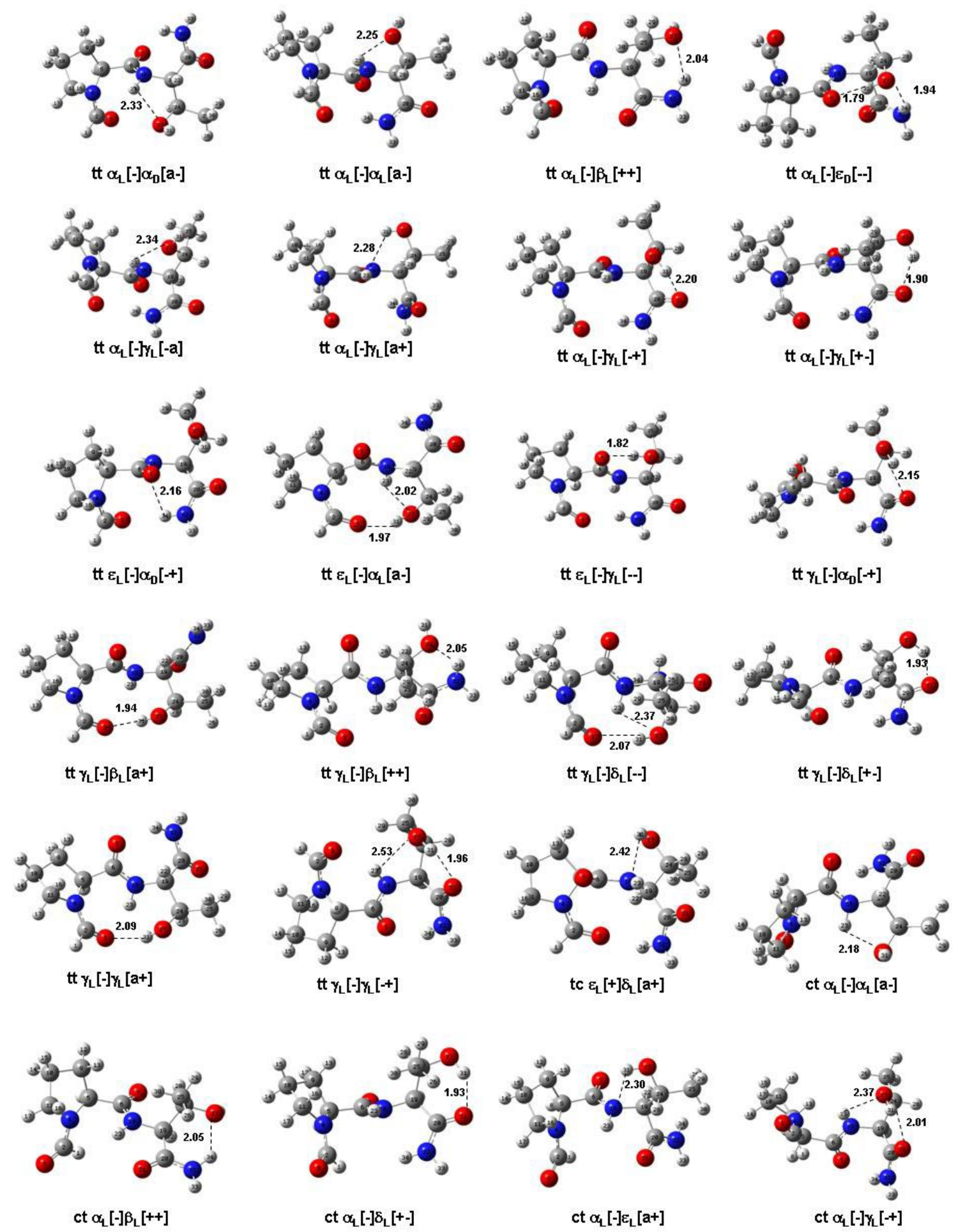

Figure F. Optimized conformations of the $30 \mathrm{HCO}-\mathrm{Pro}-\mathrm{Thr}-\mathrm{NH}_{2}$ conformations computed at the B3LYP/6-31G(d) level of theory. 


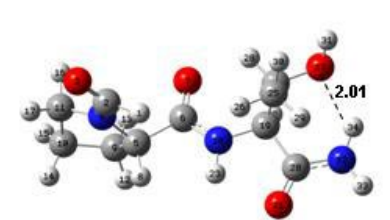

ct $\varepsilon_{\mathrm{L}}[-] \beta_{\mathrm{L}}[+\mathrm{a}]$

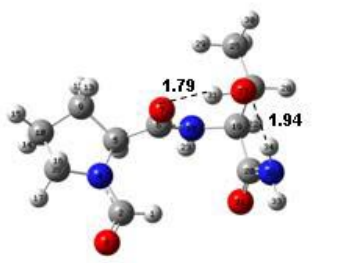

ct $\varepsilon_{\mathrm{L}}[-] \varepsilon_{\mathrm{L}}[--]$

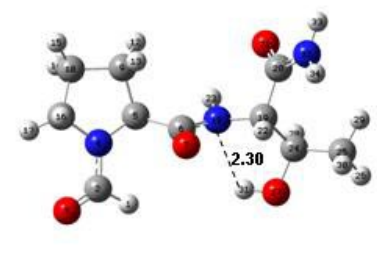

ct $\varepsilon_{\mathrm{L}}[-] \varepsilon_{\mathrm{L}}[\mathrm{a}+]$

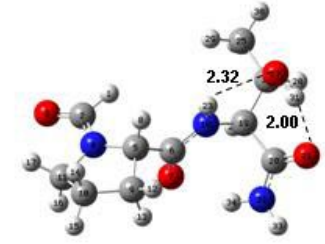

ct $\varepsilon_{\mathrm{L}}[-] \mathrm{y}_{\mathrm{L}}[-+]$

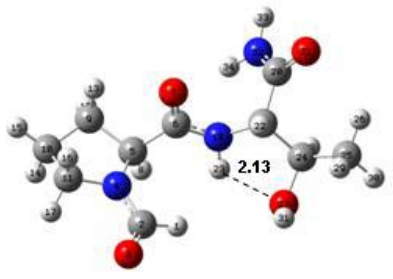

ct $y_{L}[-] o_{L}[a-]$

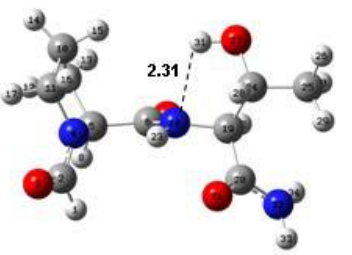

ct $y_{L}[-] \varepsilon_{L}[a+]$

Figure F. (cont'd): Optimized conformations of the $30 \mathrm{HCO}-\mathrm{Pro}-\mathrm{Thr}-\mathrm{NH}_{2}$ conformations computed at the B3LYP/6-31G(d) level of theory. 

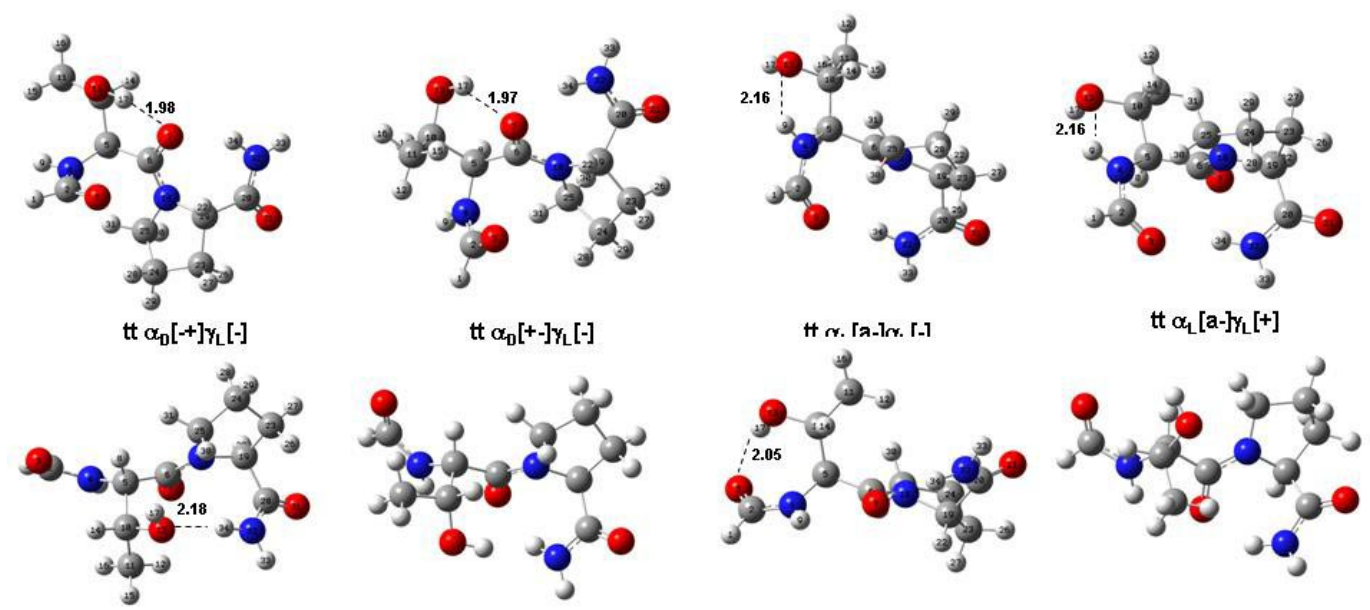

tt $\beta_{L}[++] \alpha_{L}[-]$

tt $\beta_{\mathrm{L}}[-+] \mathrm{y}_{\mathrm{L}}[-]$

tt $\beta_{L}[+-] y_{L}[+]$

tt $\beta_{\mathrm{L}}[++] y_{\mathrm{L}}[+]$

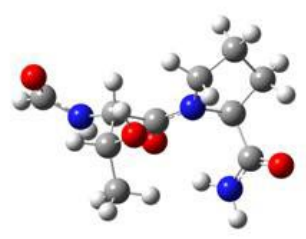

tt $\mathrm{\beta}_{\mathrm{L}}[++] \mathrm{y}_{\mathrm{L}}[-]$

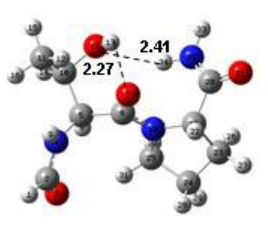

tt $\delta_{L}[+-] \alpha_{L}[-]$

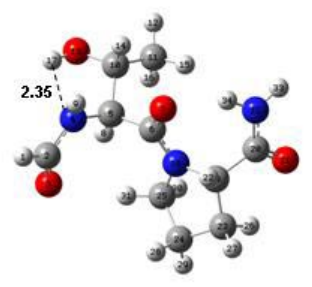

tt $\varepsilon_{\mathrm{L}}[\mathrm{a}+] y_{\mathrm{L}}[-]$

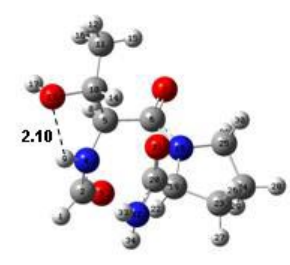

tc $\alpha_{\mathrm{L}}[\mathrm{a}-] \varepsilon_{\mathrm{L}}[-]$

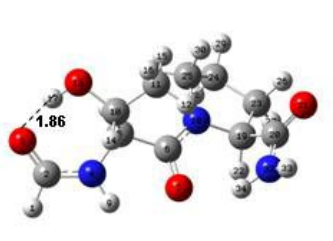

tt $\beta_{L}[a+] y_{L}[-]$

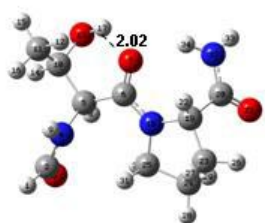

$$
\text { tt } \delta_{\mathrm{L}}[+-] \mathrm{y}_{\mathrm{L}}[+]
$$

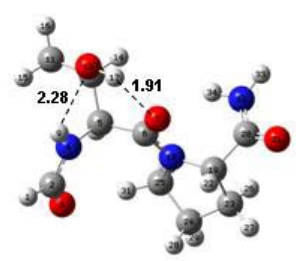

tt $y_{L}[-+] \alpha_{L}[-]$

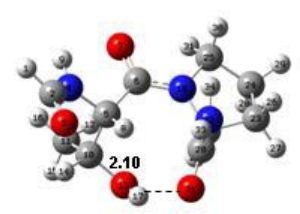

tc $\beta_{\mathrm{L}}[++] \alpha_{\mathrm{L}}[-]$
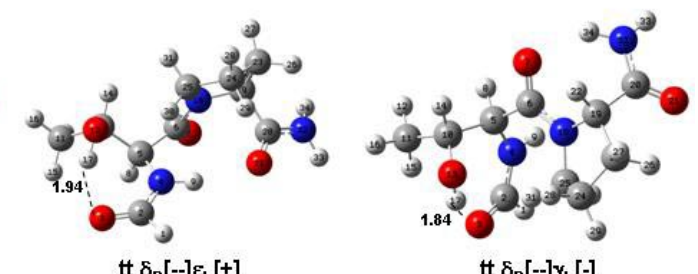

tt $\delta_{0}[-] \varepsilon_{L}[+]$

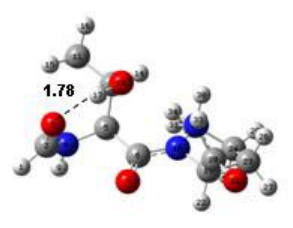

tt $\varepsilon_{0}[--] \alpha_{L}[-]$

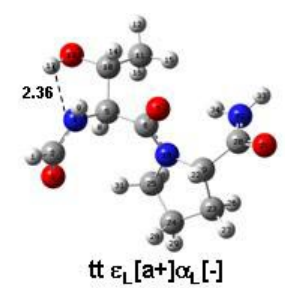

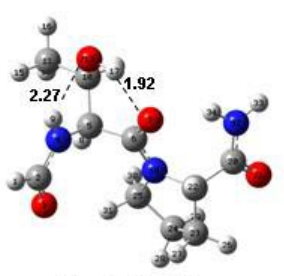

tt $y_{L}[-+] y_{L}[+]$

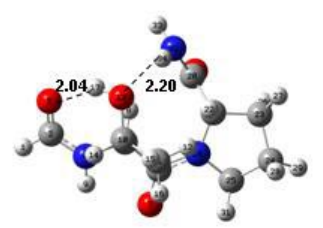

tc $\beta_{L}[a+] \varepsilon_{L}[-]$

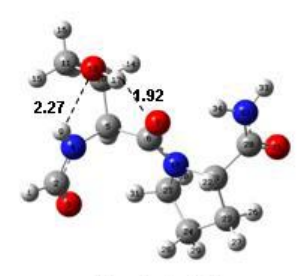

tt $y_{L}[-+] y_{L}[-]$

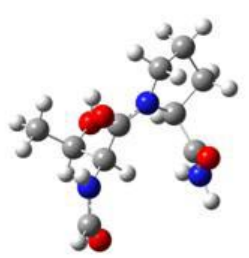

tc $\beta_{\mathrm{L}}[+-] \varepsilon_{\mathrm{L}}[-]$

Figure G. Optimized conformations of the $34 \mathrm{HCO}-\mathrm{Thr}-\mathrm{Pro}-\mathrm{NH}_{2}$ conformations computed at the B3LYP/6-31G(d) level of theory. 


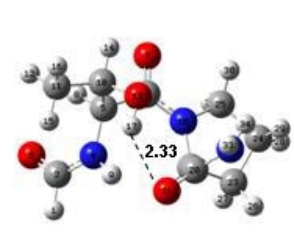

tc $\alpha_{\mathrm{L}}[\mathrm{a}-\mathrm{a}] \varepsilon_{\mathrm{L}}[-]$

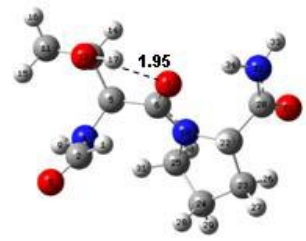

ct $\alpha_{0}[-+] y_{L}[-]$

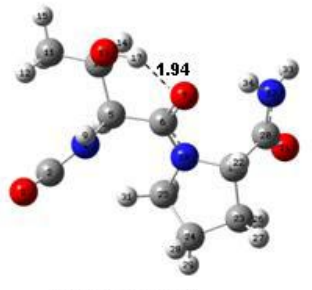

ct $\delta_{\mathrm{L}}[-+] \mathrm{y}_{\mathrm{L}}[-]$

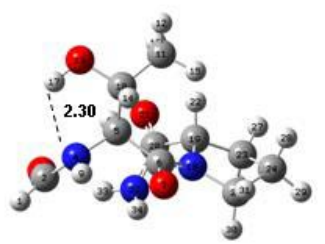

tc $\varepsilon_{\mathrm{L}}[\mathrm{a}+] \alpha_{\mathrm{L}}[-]$

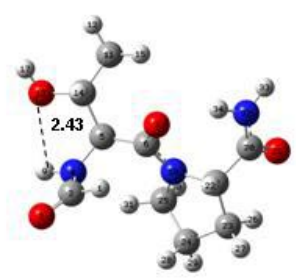

ct $\alpha_{0}[\mathrm{aa}] y_{\mathrm{L}}[-]$

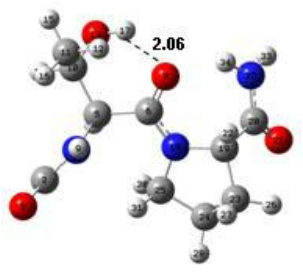

ct $\delta_{L}[+-] y_{L}[-]$

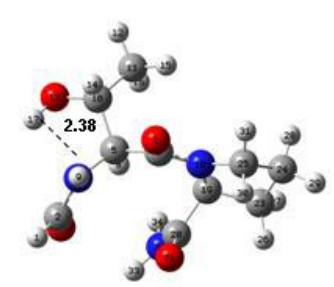

tc $\varepsilon_{\mathrm{L}}[\mathrm{a}+] \varepsilon_{\mathrm{L}}[-]$

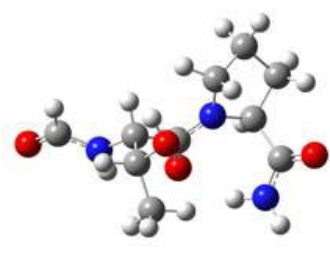

$\operatorname{ct} \beta_{L}[++] y_{L}[-]$

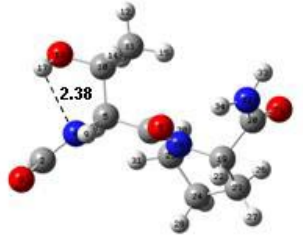

ct $\beta_{\mathrm{L}}\left[\mathrm{a}+\mathrm{y}_{\mathrm{L}}[-]\right.$

Figure G. (cont'd): Optimized conformations of the 34 HCO-Thr-Pro- $\mathrm{NH}_{2}$ conformations computed at the B3LYP/6-31G(d) level of theory. 


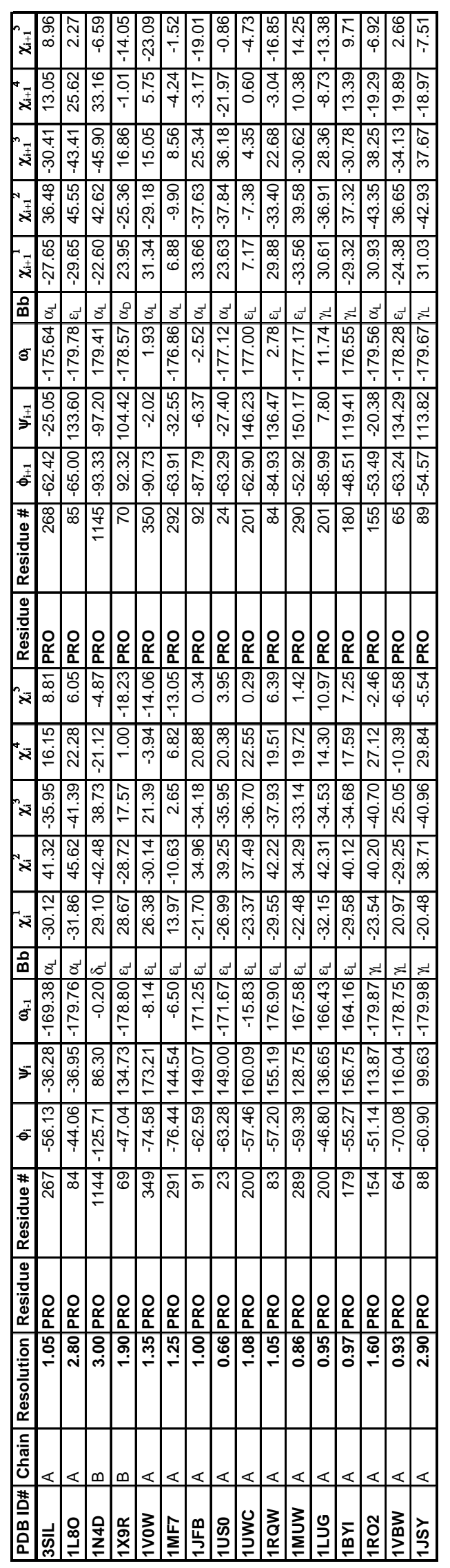

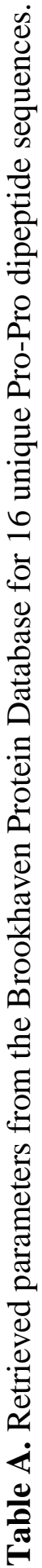




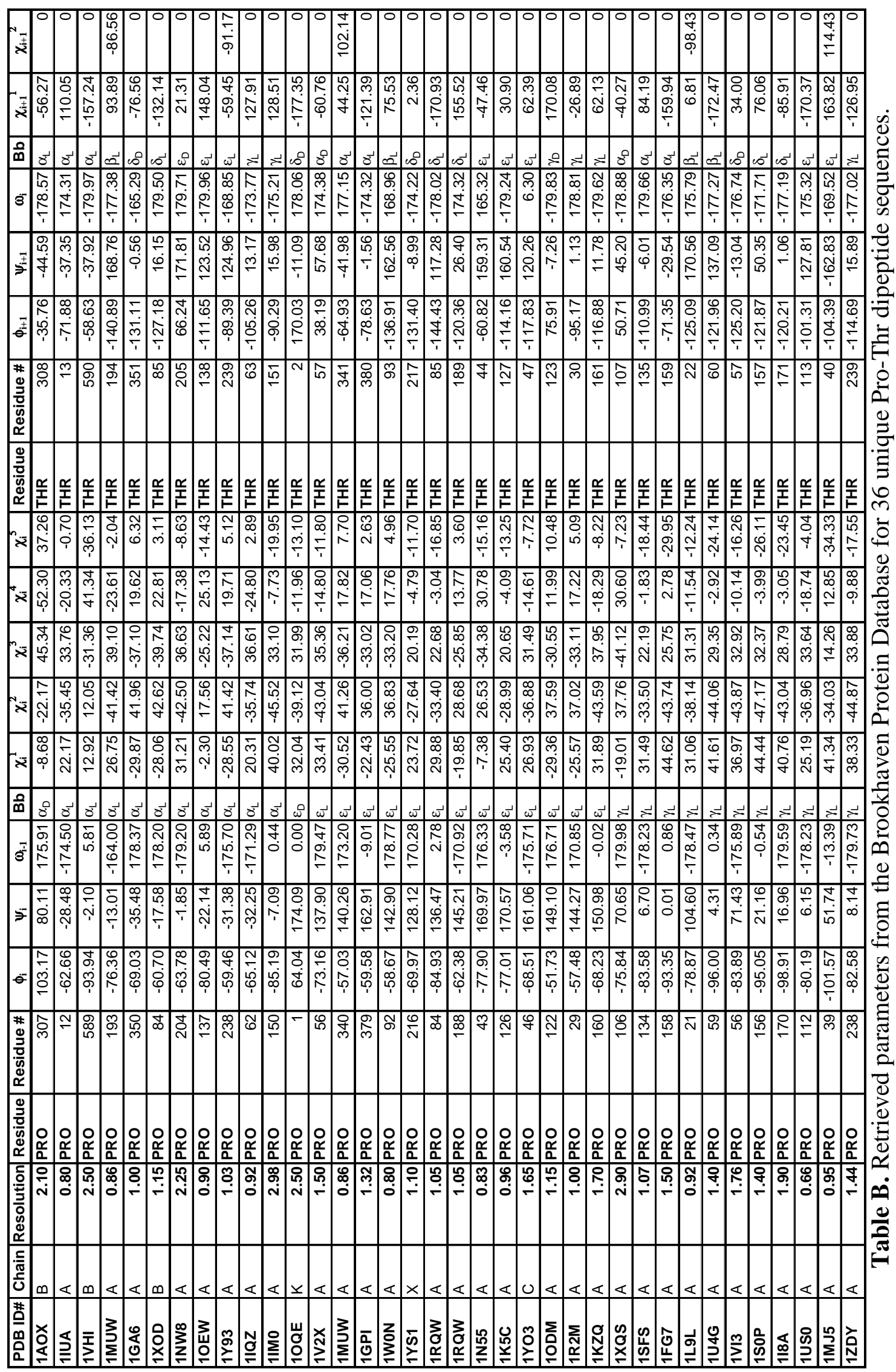




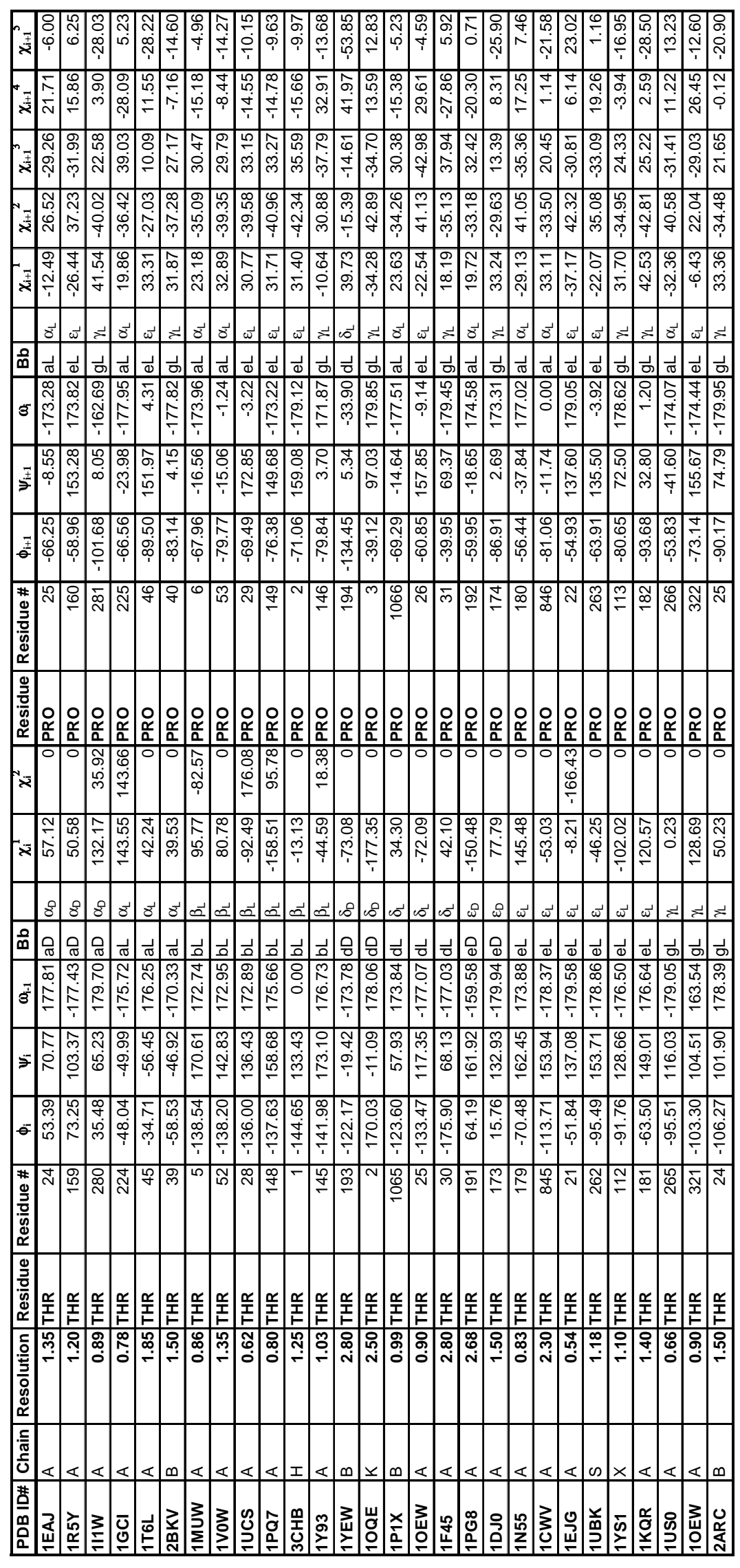




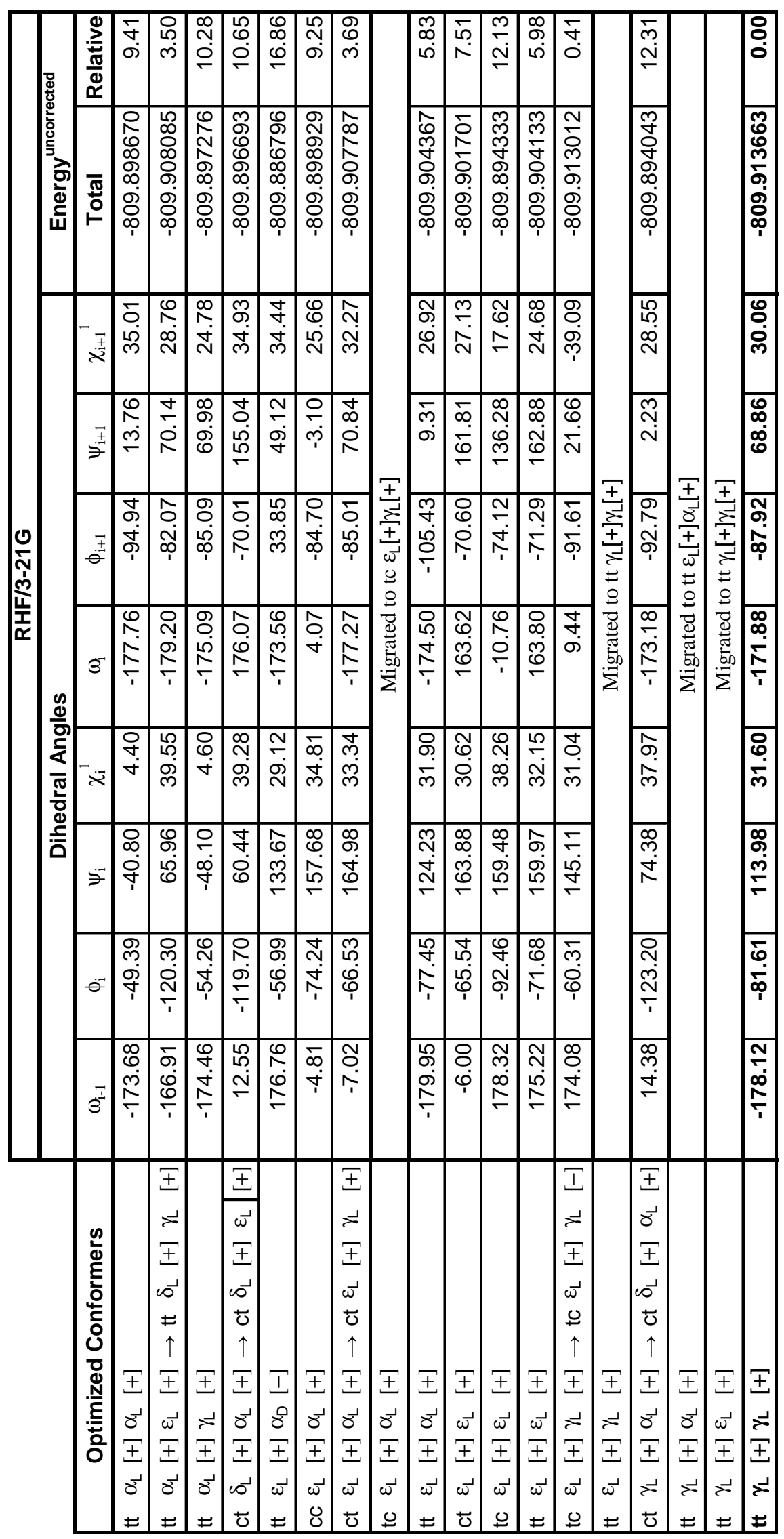

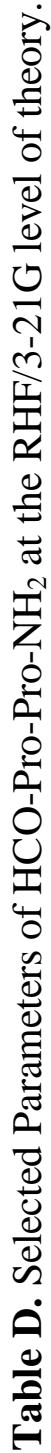




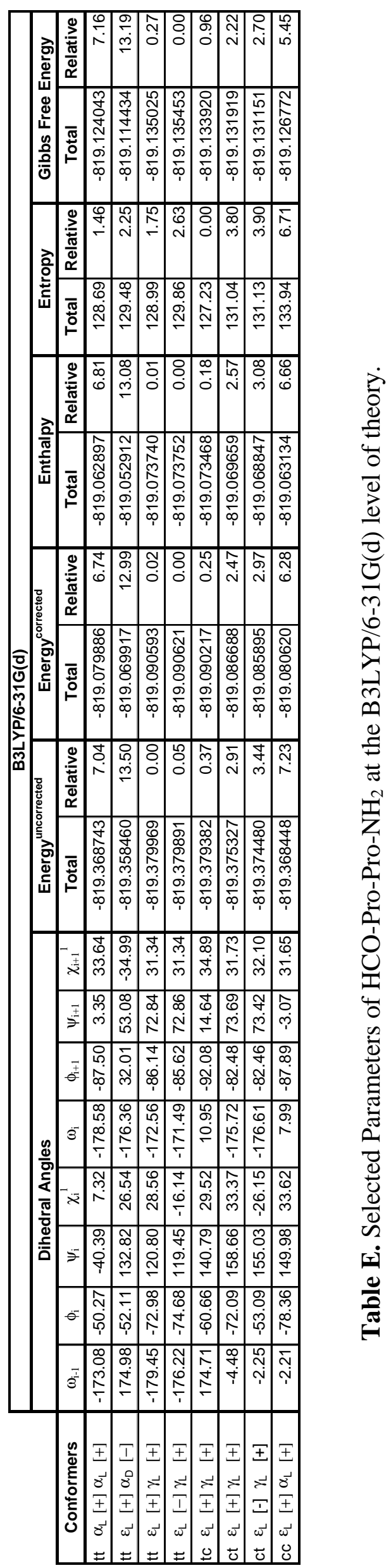




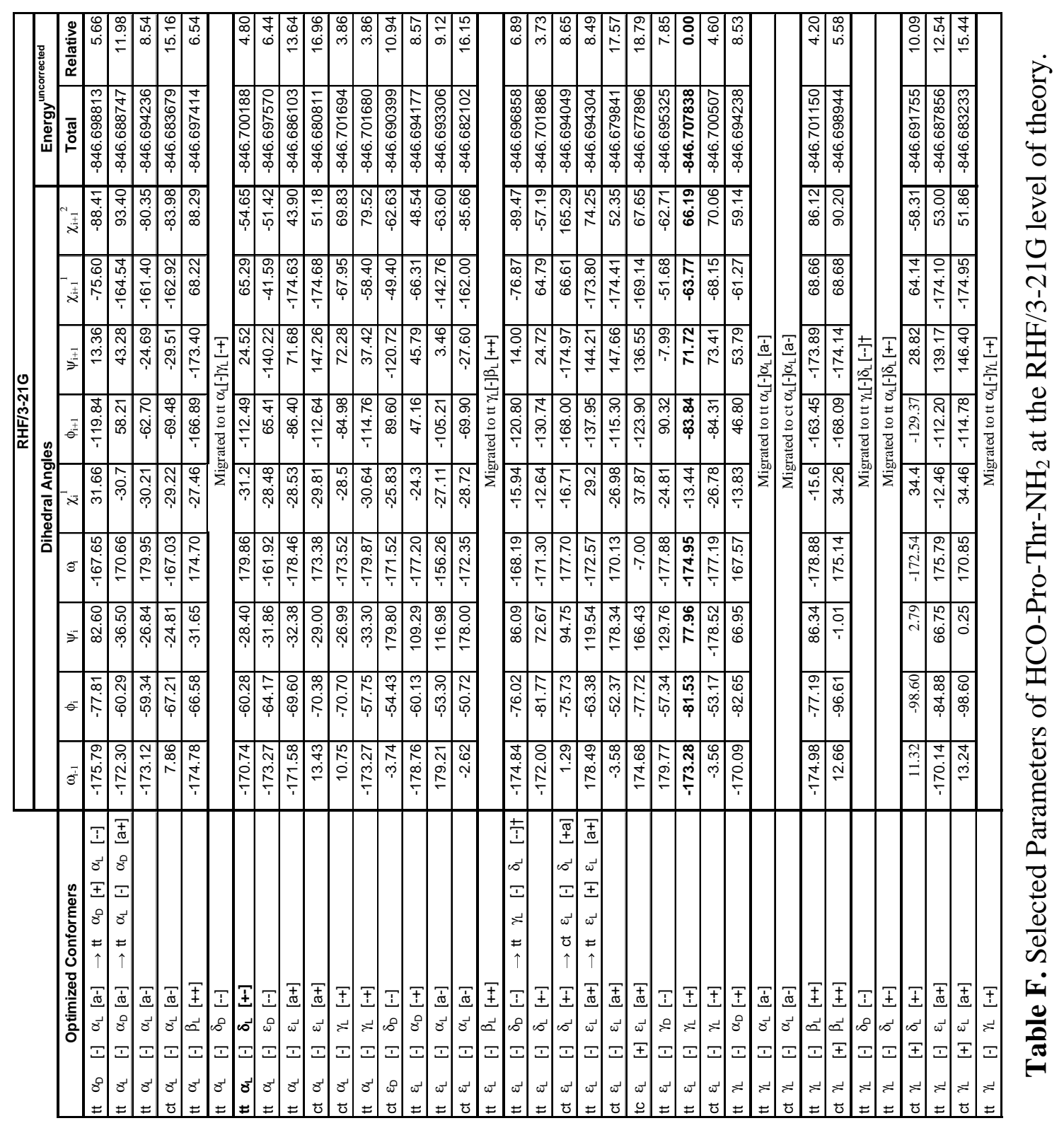




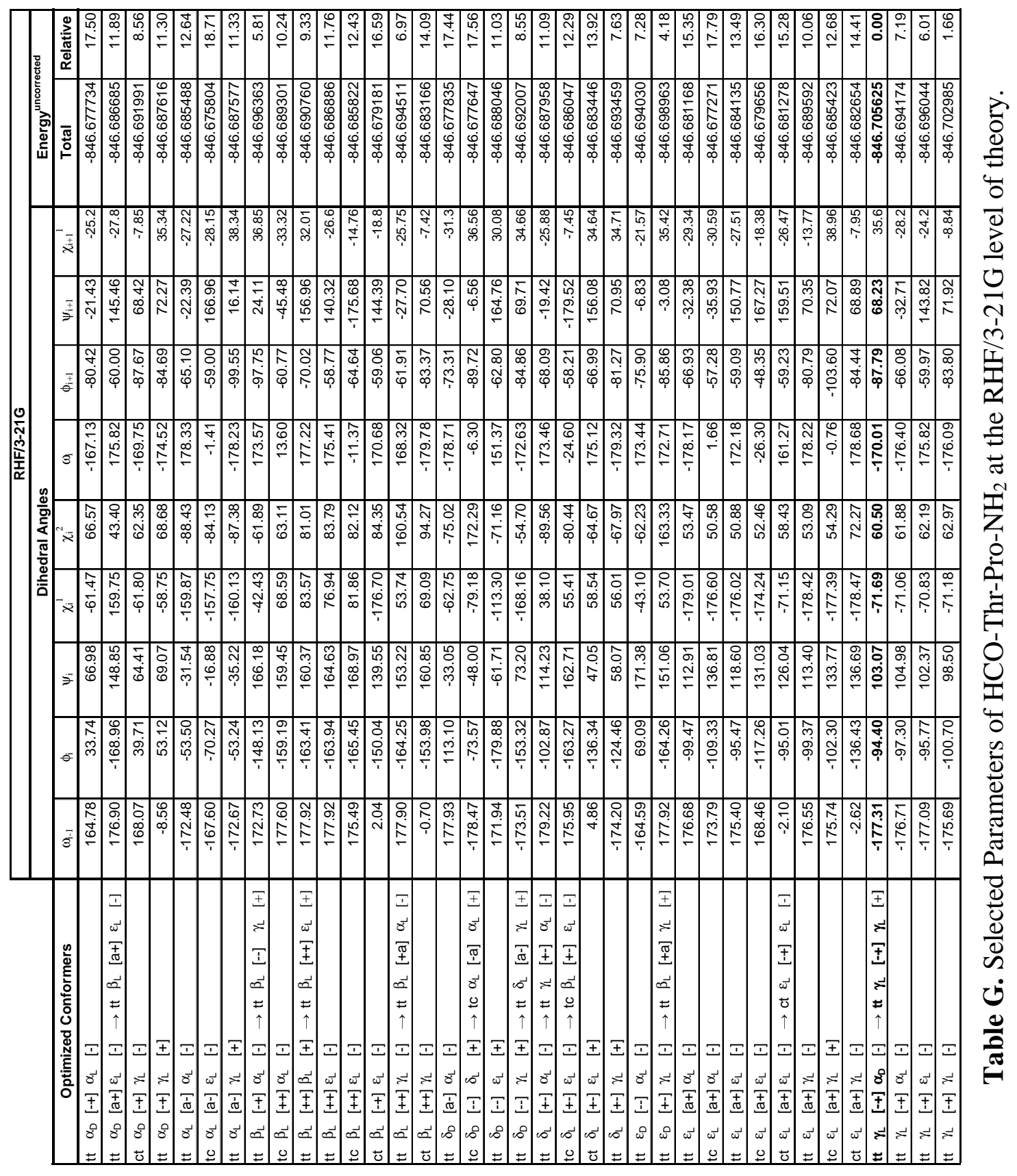




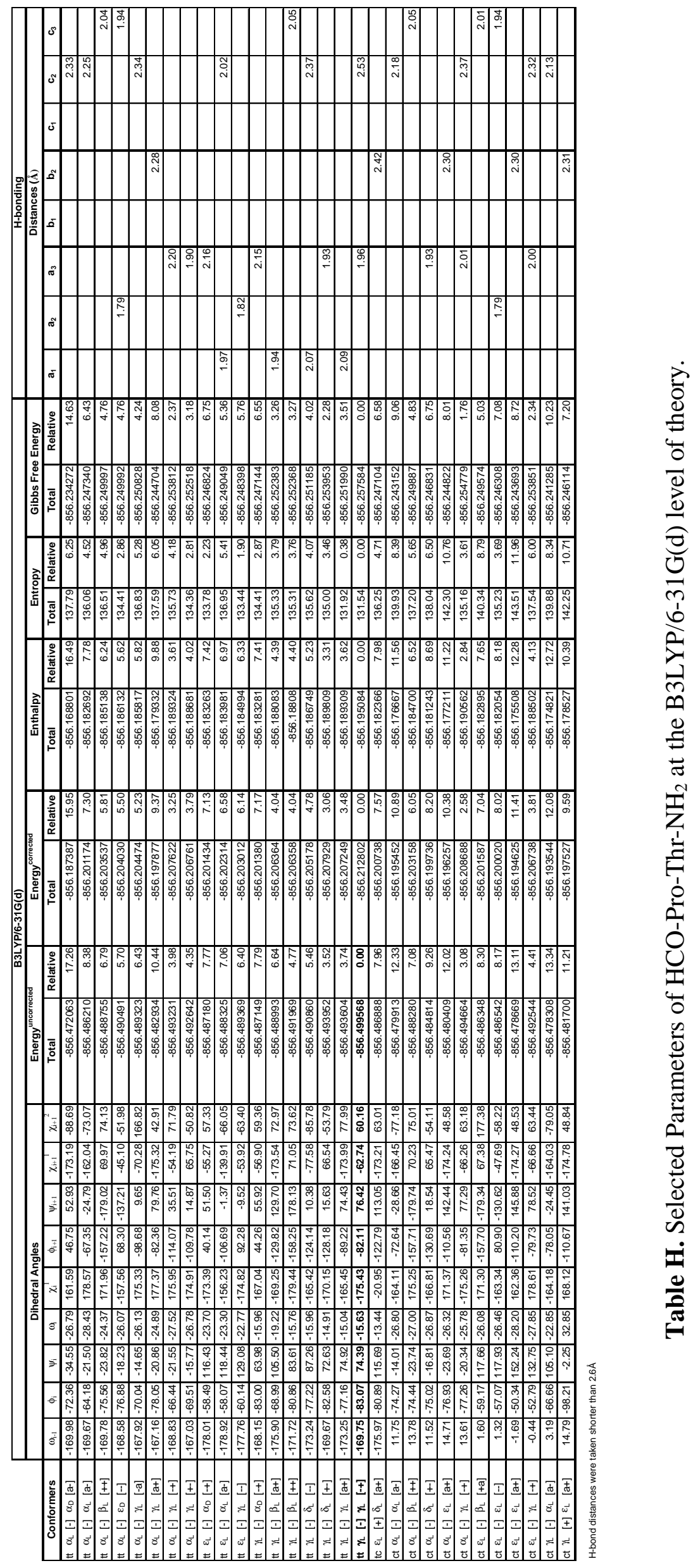




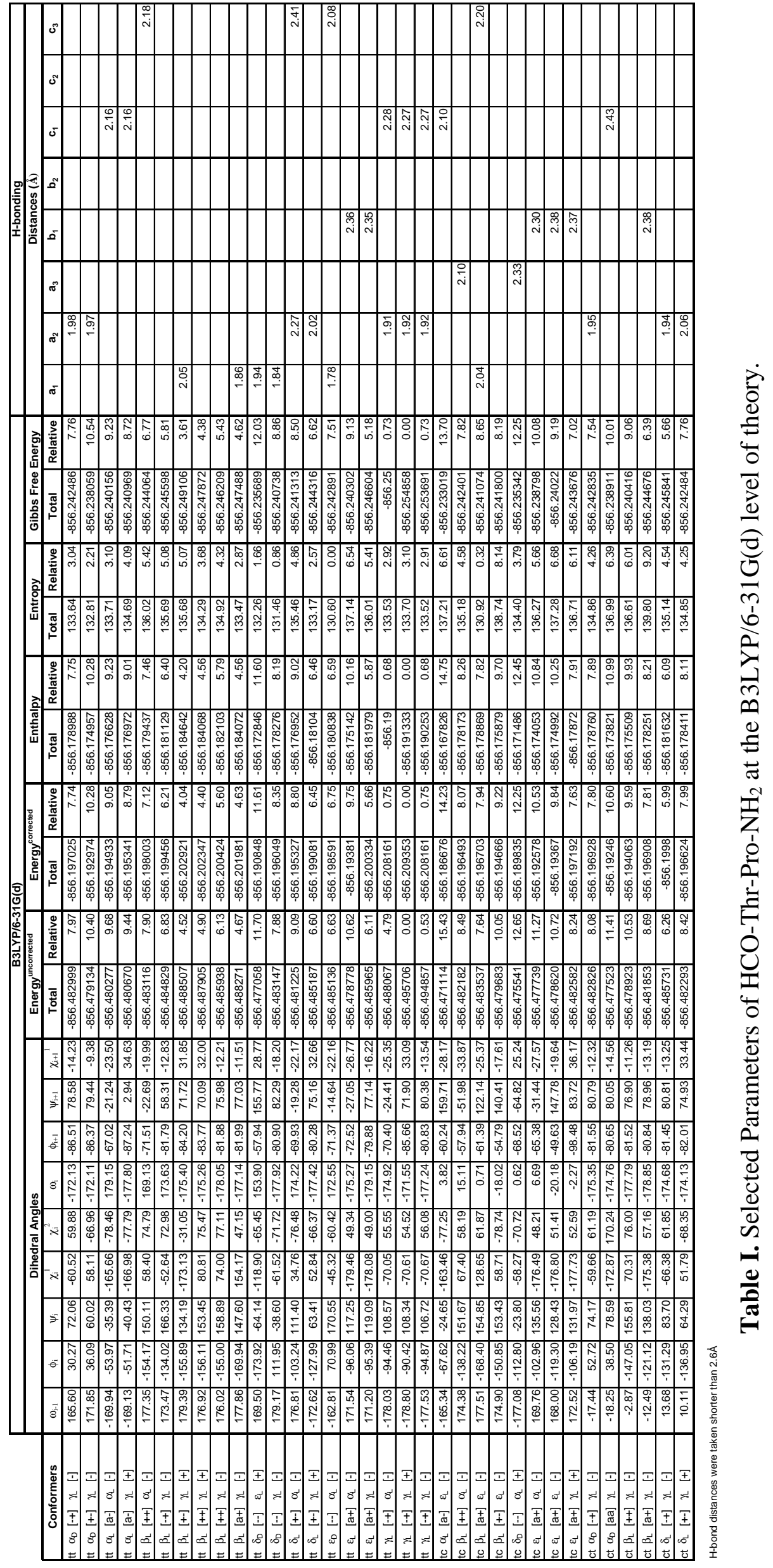




\begin{tabular}{|c|c|c|c|}
\hline HCO-Pro-Pro-NH ${ }_{2}$ & HCO- & ro-Thr-NH & HCO-Thr-Pro- $\mathrm{NH}_{2}$ \\
\hline ct $\delta_{\mathrm{L}}[++] \alpha_{\mathrm{L}}[+] \rightarrow$ ct $\varepsilon_{\mathrm{L}}[+]$ & 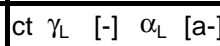 & $\rightarrow$ ct $\alpha_{\mathrm{L}}[-] \quad \alpha_{\mathrm{L}}[\mathrm{a}-]$ & ct $\varepsilon_{\mathrm{L}}[\mathrm{a}+] \quad \gamma_{\mathrm{L}}[-] \quad \rightarrow$ ct $\alpha_{\mathrm{D}}[\mathrm{aa}]$ \\
\hline ct $\varepsilon_{\mathrm{L}}[+] \quad \alpha_{\mathrm{L}}[+] \rightarrow$ ct $\varepsilon_{\mathrm{L}}[+] \gamma_{\mathrm{L}}[+]$ & {$[-] \quad \beta_{\llcorner}[++$} & $\rightarrow$ ct $\alpha_{\mathrm{L}}[-] \quad \beta_{\mathrm{L}}[++]$ & tt $\quad \alpha_{D}[-+] \quad \alpha_{L}[-] \quad \rightarrow t t \quad \alpha_{D}[-+]$ \\
\hline ct $\varepsilon_{\mathrm{L}}[+] \varepsilon_{\mathrm{L}}[+] \rightarrow$ ct $\varepsilon_{\mathrm{L}}[+] \gamma_{\mathrm{L}}[+]$ & {$[-] \quad \delta_{L}[+-]$} & $\rightarrow$ ct $\alpha_{\mathrm{L}}[-] \quad \delta_{\mathrm{L}}[+-]$ & 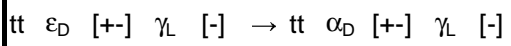 \\
\hline ct $\gamma_{\mathrm{L}}[+] \alpha_{\mathrm{L}}[+] \rightarrow$ ct $\varepsilon_{\mathrm{L}}[-] \quad \gamma_{\mathrm{L}}[+]$ & $\alpha_{\mathrm{L}}[\mathrm{a}-]$ & $\rightarrow \mathrm{tt} \quad \alpha_{\mathrm{L}}[-]$ & ct $\varepsilon_{\mathrm{L}}[\mathrm{a}+] \quad \varepsilon_{\mathrm{L}}[-] \quad \rightarrow$ ct $\beta_{\mathrm{L}}[\mathrm{a}+]$ \\
\hline tc $\varepsilon_{\mathrm{L}}[+] \alpha_{\mathrm{L}}[+] \rightarrow$ tc $\varepsilon_{\mathrm{L}}[+] \gamma_{\mathrm{L}}[+]$ & tt $\quad \alpha_{\mathrm{L}}\left[\begin{array}{lll}-] & \delta_{\mathrm{D}} & {[-\mathrm{a}]}\end{array}\right.$ & $\rightarrow \mathrm{tt} \quad \alpha_{\mathrm{L}} \quad[-]$ & tc $\beta_{\llcorner}[++] \varepsilon_{\llcorner}[-] \rightarrow$ tc $\beta_{\llcorner}[\mathrm{a}+]$ \\
\hline tc $\varepsilon_{\mathrm{L}}[+] \varepsilon_{\mathrm{L}}[+] \rightarrow$ tc $\varepsilon_{\mathrm{L}}[+] \gamma_{\mathrm{L}}[+]$ & $\operatorname{tt} \quad \alpha_{\mathrm{L}} \quad[-] \quad \delta_{\mathrm{L}}$ & $\rightarrow \mathrm{tt} \quad \alpha_{\mathrm{L}} \quad[-] \quad \gamma_{\mathrm{L}} \quad[+-]$ & tc $\delta_{\mathrm{L}} \quad[+-] \quad \varepsilon_{\mathrm{L}}[-] \quad \rightarrow$ tc $\beta_{\mathrm{L}}[+-] \quad \varepsilon_{\mathrm{L}}[-]$ \\
\hline tt $\quad \alpha_{\mathrm{L}}[+] \quad \varepsilon_{\mathrm{L}} \quad[+] \rightarrow$ tt $\quad \varepsilon_{\mathrm{L}}[+] \quad \gamma_{\mathrm{L}}[+]$ & $\alpha_{\mathrm{L}}[-] \quad \varepsilon_{\mathrm{L}} \quad[\mathrm{a}+$ & $\rightarrow \mathrm{tt} \quad \alpha_{\mathrm{L}} \quad[-] \quad \gamma_{\mathrm{L}}[\mathrm{a}+]$ & 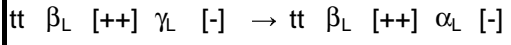 \\
\hline tt $\quad \varepsilon_{\mathrm{L}}[+] \quad \alpha_{\mathrm{L}}[+] \rightarrow$ tt $\quad \varepsilon_{\mathrm{L}}[+] \quad \gamma_{\mathrm{L}}[+]$ & {$[-] \quad \delta_{\llcorner} \quad[+-$} & $\rightarrow$ ct $\varepsilon_{\mathrm{L}} \quad[-] \quad \beta_{\mathrm{L}}[+\mathrm{a}]$ & tt $\alpha_{\mathrm{D}}[\mathrm{a}+] \varepsilon_{\mathrm{L}}[-] \quad \rightarrow \mathrm{tt} \quad \beta_{\mathrm{L}}[\mathrm{a}+] \gamma_{\mathrm{L}} \quad[-]$ \\
\hline tt $\varepsilon_{\mathrm{L}}[+] \quad \varepsilon_{\mathrm{L}}[+] \rightarrow$ tt $\varepsilon_{\mathrm{L}}[+] \gamma_{\mathrm{L}}[+]$ & ct $\varepsilon_{\mathrm{D}}[-] \quad \delta_{\mathrm{D}}[--]$ & $\rightarrow$ ct $\varepsilon_{\mathrm{L}}[-] \quad \varepsilon_{\mathrm{L}}[--]$ & tt $\beta_{\mathrm{L}}[+++] \beta_{\mathrm{L}}[+] \rightarrow \mathrm{tt} \quad \beta_{\mathrm{L}}[++] \gamma_{\mathrm{L}}[+]$ \\
\hline tt $\quad \gamma_{\mathrm{L}} \quad[+] \alpha_{\mathrm{L}}[+] \rightarrow \mathrm{tt} \quad \varepsilon_{\mathrm{L}}[+] \quad \gamma_{\mathrm{L}}[+]$ & {$[+] \varepsilon_{\mathrm{L}}[\mathrm{a}+$} & $\rightarrow$ tc $\varepsilon_{\mathrm{L}}[+] \delta_{\mathrm{L}}[\mathrm{a}+$ & 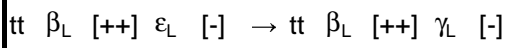 \\
\hline 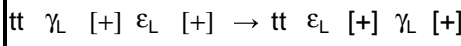 & tt $\quad \alpha_{D}[-] \quad \alpha_{L}[a-]$ & $\rightarrow \mathrm{tt} \quad \varepsilon_{\mathrm{L}} \quad[-] \quad \alpha_{\mathrm{L}}[\mathrm{a}-]$ & $\mathrm{tt} \quad \beta_{\mathrm{L}}\left[-[-+] \quad \alpha_{\mathrm{L}}[-] \quad \rightarrow \mathrm{tt} \quad \beta_{\mathrm{L}}[-++]\right.$ \\
\hline 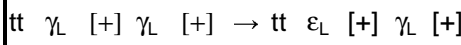 & {$[-] \gamma_{D}[--]$} & $\rightarrow \mathrm{tt} \quad \varepsilon_{\mathrm{L}} \quad[-] \quad \gamma_{\mathrm{L}}[-[-]$ & $\gamma_{\mathrm{L}}[++] \rightarrow$ tt $\beta_{\mathrm{L}}[+-]$ \\
\hline 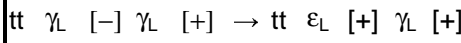 & {$[-] \quad \alpha_{L}[a-]$} & $\rightarrow$ ct $\gamma_{\mathrm{L}} \quad[-] \quad \alpha_{\mathrm{L}}[\mathrm{a}-]$ & $\delta_{\mathrm{L}}[+] \rightarrow$ tc $\delta_{\mathrm{D}}[--]$ \\
\hline 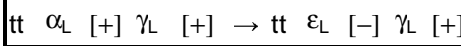 & {$[-] \quad \beta_{\llcorner}[++$} & $\rightarrow \mathrm{tt} \quad \gamma_{\mathrm{L}} \quad[-] \quad \beta_{\mathrm{L}}[++]$ & 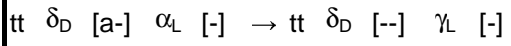 \\
\hline & $\begin{array}{lllll}\mathrm{tt} & \varepsilon_{\mathrm{L}} & {[-]} & \delta_{\mathrm{D}} & {[--]}\end{array}$ & $\rightarrow \mathrm{tt} \quad \gamma_{\mathrm{L}} \quad[-] \quad \delta_{\mathrm{L}} \quad[--]$ & ct $\beta_{\mathrm{L}}[-+] \quad \varepsilon_{\mathrm{L}} \quad[-] \quad \rightarrow$ ct $\delta_{\mathrm{L}}[-+]$ \\
\hline & tt $\varepsilon_{\mathrm{L}} \quad[-] \quad \delta_{\mathrm{L}}[+-]$ & $\rightarrow \mathrm{tt} \quad \gamma_{\mathrm{L}} \quad[-] \quad \delta_{\mathrm{L}}[+-]$ & ct $\delta_{\mathrm{L}}[++-] \quad \varepsilon_{\mathrm{L}}[+] \rightarrow$ ct $\delta_{\mathrm{L}}[+-] \quad \gamma_{\mathrm{L}}[+]$ \\
\hline & 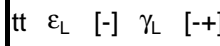 & $\rightarrow \mathrm{tt} \quad \gamma_{\mathrm{L}} \quad[-] \quad \gamma_{\mathrm{L}}[-+]$ & tt $\varepsilon_{\mathrm{L}}[\mathrm{a}+] \quad \varepsilon_{\mathrm{L}}[-] \quad \rightarrow$ tt $\varepsilon_{\mathrm{L}}[\mathrm{a}+] \quad \gamma_{\mathrm{L}}$ \\
\hline & $\begin{array}{lllll}\text { tt } & \gamma_{\mathrm{L}} & {[-]} & \varepsilon_{\mathrm{L}} & {[\mathrm{a}+}\end{array}$ & $\rightarrow \mathrm{tt} \quad \gamma_{\mathrm{L}}[-] \quad \gamma_{\mathrm{L}}[\mathrm{a}+]$ & tt $\quad \alpha_{D}\left[\begin{array}{llllllll} & \gamma_{\mathrm{L}} & {[+]} & \rightarrow \mathrm{tt} & \gamma_{\mathrm{L}} & {[-+]} & \gamma_{\mathrm{L}} & {[+]}\end{array}\right.$ \\
\hline & & & tt $\gamma_{\mathrm{L}}\left[-[-] \quad \alpha_{\mathrm{D}}[-] \quad \rightarrow\right.$ tt $\gamma_{\mathrm{L}}[-++] \gamma_{\mathrm{L}}$ \\
\hline & & & 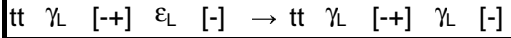 \\
\hline
\end{tabular}

Table G. A list of conformations that converged away from their initial geometries at the B3LYP/6-31G(d) level of theory for the three dipeptides. 